\title{
Apatinib combined with paclitaxel-based chemotherapy in patients with taxane-resistant advanced gastric cancer: a single-arm exploratory study
}

\author{
Shen Zhao ${ }^{1,2,3}$, Nanfeng Fan ${ }^{1,2}$, Hui Li ${ }^{1,2}$, Jie Liu ${ }^{1,2}$, Feng Huang ${ }^{1,2}$, Yigui Chen ${ }^{1,2}$, Min Zhou ${ }^{1,2}$, \\ Jiaqing Yu ${ }^{1,2}$, Rongbo Lin ${ }^{1,2,3}$ \\ ${ }^{1}$ Department of Gastrointestinal Medical Oncology, Fujian Cancer Hospital, Fuzhou, Fujian, China; ${ }^{2}$ Fujian Medical University Cancer Hospital, \\ Fuzhou, Fujian, China; ${ }^{3}$ Fujian Key Laboratory of Translational Cancer Medicine, Fuzhou, Fujian, China \\ Contributions: (I) Conception and design: R Lin; (II) Administrative support: R Lin; (III) Provision of study materials or patients: S Zhao, N Fan, H \\ Li, J Liu, Y Chen, J Yu, F Huang, R Lin; (IV) Collection and assembly of data: M Zhou, J Yu; (V) Data analysis and interpretation: S Zhao, R Lin; (VI) \\ Manuscript writing: All authors; (VII) Final approval of manuscript: All authors \\ Correspondence to: Prof. Rongbo Lin. Fujian Cancer Hospital, Fuzhou, Fujian, China. Email: linrongbo@fjzlhospital.com.
}

Background: Apatinib combined with chemotherapy might be effective and safe for the management of advanced gastric cancer, but the available data are limited. To investigate the efficacy and safety of apatinib in combination with paclitaxel (PTX) alone or POF (PTX, oxaliplatin, and 5-fluorouracil) in patients with taxane-resistant advanced gastric cancer.

Methods: Patients with taxane-resistant advanced gastric cancer were enrolled in the single-center, openlabeled, single-arm, exploratory study (ClinicalTrials.gov \#NCT02697838). Apatinib was administered at $850 \mathrm{mg}$ po in combination with weekly PTX or the POF regimen. The primary endpoint was the objective response rate (ORR). The secondary endpoints included disease control rate (DCR), progression-free survival (PFS), overall survival (OS), the time to tumor progression (TTP), and safety.

Results: Twenty participants were recruited from 08/2016 to $01 / 2018$. The duration of the study treatment was $2.07(0.03-16.2)$ months. The median follow-up was $24.8(0.3-26.0)$ months. The reasons for termination of treatment were disease progression $(n=6)$, adverse events (AEs) ( $n=5)$, and patients' will $(\mathrm{n}=9)$. The ORR was $11.1 \%$ (95\% CI: $1.4-34.7 \%)$ and the DCR was $77.8 \%$ (95\% CI: 52.4-93.6\%). The median PFS was 3.5 (95\% CI: 1.9-5.1) months, the median OS was 4.7 (95\% CI: 2.0-7.3) months, and the median TTP was 4.2 (95\% CI: 0.562-7.838) months. All 20 (100\%) patients had AEs, 17 (85\%) had apatinib treatment-emergent AEs (TEAEs), and 18 (90\%) had chemotherapy TEAEs. The main grade 3-4 TEAEs were neutropenia, leukopenia, hypertension, and anemia.

Conclusions: This preliminary study suggests that apatinib combined with PTX or POF might be effective and tolerable in patients with chemotherapy-refractory gastric cancer. Studies are necessary to confirm the results.

Trial registration: ClinicalTrials.gov \#NCT02697838.

Keywords: Apatinib; paclitaxel (PTX); drug resistance; advanced stage; gastric cancer.

Submitted Jul 27, 2020. Accepted for publication Oct 09, 2020.

doi: $10.21037 / \mathrm{atm}-20-5841$

View this article at: http://dx.doi.org/10.21037/atm-20-5841 


\section{Introduction}

Gastric adenocarcinomas are tumors of the stomach, including tumors of the noncardia and the subcardia (Siewert type III), with center starting 2-5 $\mathrm{cm}$ below the esophagogastric junction (EGJ) $(1,2)$. The estimated global incidence of gastric cancer was 1,033,701 cases in 2018 (3). The incidence of gastric cancer is the highest in Eastern Asia, Eastern Europe, and South America $(2,4)$. More men are twice more affected than women (2). The direct cause of gastric cancer is not clear, but Helicobacter pylori infection and some hereditary cancer predisposition syndromes may play a role $(1,2,4)$. Patients often present with nonspecific symptoms, which may include anorexia, weight loss, abdominal pain, dyspepsia, vomiting, and early satiety $(2,4)$. The 5 -year survival of patients with gastric cancer is $67 \%$ for localized disease, $31 \%$ for regional disease, and $5 \%$ for distant disease (5).

The management of gastric cancer includes surgery, chemotherapy, and radiation therapy (1). Chemotherapy is the standard treatment for advanced gastric cancer, and taxanes are commonly used chemotherapy drugs. Firstline treatment scheme includes docetaxel (DOC)/paclitaxel (PTX) + fluorouracil (FU) (1). In addition, the FNF-004 trial showed that the POF regimen (PTX + oxaliplatin $+\mathrm{FU})$ as the first-line treatment for advanced gastric cancer led to a higher progression-free survival (PFS) than FOLFOX, and was tolerable (6). Second-line treatment mainly includes DOC or PTX monotherapy (1), but after failing to taxane-based regimens or PTX alone, patients have few other treatment options.

Apatinib is an orally bioavailable tyrosine kinase inhibitor (TKI) that selectively inhibits the vascular endothelial growth factor receptor (VEGFR)-2 (and also mildly inhibits c-Kit and c-SRC tyrosine kinases) and angiogenesis in cancer cells (7). Apatinib inhibits VEGFmediated endothelial cell migration and proliferation, blocking new blood vessel formation in tumors (7). Apatinib might improve overall and PFS in patients with advanced or metastatic gastric or gastroesophageal junction adenocarcinoma refractory to $\geq 2$ chemotherapy regimens $(8,9)$. A prospective study showed that apatinib in combination with chemotherapy was effective and tolerable in the treatment of advanced chemotherapy-refractory gastric cancer, and had higher PFS than that of apatinib alone (10). Nevertheless, because of the different first- and second-line chemotherapies and different chemotherapies in combination with apatinib in the study (10), it is difficult to evaluate the efficacy and safety accurately.
Therefore, the aim of this single-arm exploratory study was to investigate the efficacy and safety of apatinib in combination with POF or PTX in patients with taxaneresistant advanced gastric cancer. The results could provide additional treatment options for patients with advanced gastric cancer. We present the following article in accordance with the TREND reporting checklist (available at http://dx.doi.org/10.21037/atm-20-5841).

\section{Methods}

\section{Study design and participants}

This was a single-center, open-labeled, single-arm, exploratory study. This study is performed in accordance with the Declaration of Helsinki (as revised in 2013), Good Clinical Practice, and related laws. The study protocol was approved by the ethics committee of Fujian Cancer Hospital. The study was registered (ClinicalTrials.gov \#NCT02697838). All patients provided a signed informed consent form before any study procedure.

The key inclusion criteria were: (I) $18-70$ years of age; (II) adenocarcinoma of the stomach or gastroesophageal junction confirmed by pathology (including histology or cytology); (III) locally advanced or metastatic gastric cancer (AGC) that cannot be resected; (IV) resistant to chemotherapy, including taxanes (definition of resistance: disease progression according to Response Evaluation Criteria In Solid Tumors (RECIST) 1.1 criteria evaluation (11) or recurrence during chemotherapy or within 3 months after chemotherapy); (V) Eastern Cooperative Oncology Group (ECOG) performance status (PS) 0-1; (VI) estimated survival time $\geq 3$ months; and (VII) sufficient organ function. The key exclusion criteria were: (I) the patients who only failed to taxanes, but have not been challenged with other anti-tumor drugs (such as fluorouracil and platinum) that may be effective for the treatment of gastric cancer; (II) poor control of hypertension; (III) a previous history of severe cardiovascular disease; (IV) abnormal coagulation or a possibility of massive hemorrhage of the digestive tract; or (V) patients with metastasis to the central nervous system.

\section{Treatment}

Apatinib was administered at $850 \mathrm{mg}$ po qd from day 1 to day 28 of every 28 -day cycles. The initial dose of patients with poor tolerance was $750 \mathrm{mg}$ po qd. For the participants receiving an initial dose of $850 \mathrm{mg} \mathrm{qd}$, dose adjustment was 
allowed twice at most, and the dose could be reduced to 750 or $500 \mathrm{mg}$ qd. When the initial dose was $750 \mathrm{mg}$ qd, dose adjustment was allowed only once at most, and the dose could be reduced to $500 \mathrm{mg}$ qd. When the dose reached $500 \mathrm{mg} \mathrm{qd}$, further dose adjustment was not allowed, but treatment suspension was still allowed.

The recommended dose of PTX in combination with apatinib was $80 \mathrm{mg} / \mathrm{m}^{2}$, and PTX was administered on days 1,8 , and 15 of every 28 -day cycle. During the treatment of PTX, if grade $>3$ hematologic toxicity occurred twice (except anemia which could be corrected by iron supplement or blood transfusion), a dose reduction by $10 \mathrm{mg} / \mathrm{m}^{2}$ to a dose of $70 \mathrm{mg} /$ $\mathrm{m}^{2}$ was recommended for the next course of treatment. If the same toxic reaction still occurred, another dose reduction by 10 $\mathrm{mg} / \mathrm{m}^{2}$ to a dose of $60 \mathrm{mg} / \mathrm{m}^{2}$ was recommended. If the same toxic reaction still occurred after the second dose reduction, it was recommended to stop the treatment and withdraw from the study. Hematological toxicity had to be restored below grade 2 before the next cycle of treatment (except anemia, leukopenia and decreased neutrophil count). Dose suspension and dose reduction were allowed only when grade $>2$ nonhematologic toxicity occurred.

For PTX + oxaliplatin + FU, the POF regimen, in combination with apatinib, was used by taxane-resistant patients as follows. Intravenous PTX was administrated at $135 \mathrm{mg} / \mathrm{m}^{2}$ over $3 \mathrm{~h}$, followed by intravenous folic acid at $400 \mathrm{mg} / \mathrm{m}^{2}$ and oxaliplatin at $85 \mathrm{mg} / \mathrm{m}^{2}$ over $2 \mathrm{~h}$; and followed by continuous intravenous infusion of $\mathrm{FU}$ at $2,400 \mathrm{mg} / \mathrm{m}^{2}$ over $46 \mathrm{~h}$. The administration of the drugs started on days 1 and 15 of each 28 -day cycle. If grade 2 peripheral neurotoxicity, grade 4 neutropenia, or grade 3/4 thrombocytopenia occurred, PTX dosage was reduced to $120 \mathrm{mg} / \mathrm{m}^{2}$, and oxaliplatin dosage was reduced to $75 \mathrm{mg} / \mathrm{m}^{2}$. If grade 3 peripheral neurotoxicity occurred, the administration of PTX and oxaliplatin was discontinued, and the patient was withdrawn from the study. If grade $3 / 4$ diarrhea or hand-foot syndrome occurred, FU dosage was reduced to $2,000 \mathrm{mg} / \mathrm{m}^{2}$.

After PTX alone was reduced to $60 \mathrm{mg} / \mathrm{m}^{2}$ (one-week plan) or PTX in the POF regimen was reduced to $120 \mathrm{mg} / \mathrm{m}^{2}$, further dose adjustment was not allowed, but the suspension was still allowed.

Treatment was continued until disease progression, unacceptable toxicity, or withdrawal of consent.

\section{Endpoints and assessment}

The primary endpoint was objective response rate (ORR), including complete response (CR) and partial response (PR) cases. RECIST 1.1 (11) was used, and imaging evaluation was conducted every 8 weeks. The secondary endpoints included disease control rate (DCR), PFS, overall survival (OS), and time to tumor progression (TTP). NCI-CTCAE 4.03 (12) was used to evaluate the safety endpoint. The patients were followed until May 13, 2019.

\section{Statistical analysis}

As this was an exploratory study, no power analysis was performed. The full analysis set (FAS) included all participants with at least one study drug administration and one efficacy evaluation, according to the principle of intention-to-treat (ITT). The safety analysis set (SS) included all participants with at least one study drug administration and the safety record after drug use.

All statistical analyses were programmed using IBM SPSS Statistics 25.0.0.1 (IBM, Armonk, NY, USA). The continuous data were presented as means, standard deviations, medians, maximum values, and minimum values. The categorical data and ordinal data were presented as frequencies (constituent ratio), rate, and confidence intervals (CI). The Clopper-Pearson (Exact) method was used to calculate the $95 \%$ CI of ORR and DCR. The Kaplan-Meier method was used to estimate the median PFS and OS and draw the survival curve. Only descriptive statistical analysis was conducted for safety evaluation.

\section{Results}

\section{Characteristics of the participants}

A total of 20 participants were recruited from August 2016 to January 2018 (Table 1). There were 12 men and 8 women. They were 54.5 (31.0-69.0) years of age. Among them, 18 participants received apatinib (850 $\mathrm{mg}$ q.d.) + POF, and two participants received apatinib (850 $\mathrm{mg}$ q.d.) + PTX. The duration of the study treatment was 2.07 (0.03-16.2) months. Two patients did not receive imaging evaluation. The median follow-up was $24.8(0.3-26.0)$ months. The reasons for termination of treatment were disease progression $(n=6)$, adverse events (AEs) $(n=5)$, and patients' will $(n=9)$. All 20 patients were included in the safety analysis, but two patients did not have a radiological examination, and 18 patients were included in the efficacy analysis. 


\section{Page 4 of 9}

Table 1 Baseline characteristics of the participants

\begin{tabular}{|c|c|}
\hline Characteristics & Total $(n=20)$ \\
\hline Age, years, median (range) & $54.5(31.0-69.0)$ \\
\hline Sex, male/female & $12 / 8$ \\
\hline \multicolumn{2}{|l|}{ ECOG PS } \\
\hline 0 & 5 \\
\hline 1 & 15 \\
\hline \multicolumn{2}{|l|}{ Tumor differentiation } \\
\hline Undifferentiated & 1 \\
\hline Poorly differentiated & 8 \\
\hline Moderate/poor differentiated & 1 \\
\hline Moderate differentiated & 6 \\
\hline N/A & 4 \\
\hline \multicolumn{2}{|l|}{ Stage } \\
\hline III & 2 \\
\hline IV & 18 \\
\hline \multicolumn{2}{|l|}{ Prior chemotherapy lines } \\
\hline 1 & 7 \\
\hline 2 & 8 \\
\hline 3 & 5 \\
\hline \multicolumn{2}{|l|}{ Metastatic lesions } \\
\hline$\leq 2$ & 12 \\
\hline$>2$ & 8 \\
\hline
\end{tabular}

ECOG, Eastern Cooperative Oncology Group; N/A, not available.

Table 2 Tumor response

\begin{tabular}{lc}
\hline & Total $(\mathrm{n}=18)$ \\
\hline Best overall response, $\mathrm{n}(\%)$ & 0 \\
Complete response & 2 \\
Partial response & 12 \\
Stable disease & 4 \\
Progressive disease & $2(11.1 \%)$ \\
ORR, $\mathrm{n}(\%)$ & $1.4-34.7 \%$ \\
$95 \% \mathrm{Cl}$ & $14(77.8 \%)$ \\
DCR, $\mathrm{n} \mathrm{( \% )}$ & $52.4-93.6 \%$ \\
$95 \% \mathrm{Cl}$ &
\end{tabular}

The Clopper-Pearson (Exact) method was used to calculate the confidence intervals of ORR and DCR. ORR, objective response rate; $\mathrm{DCR}$, disease control rate.
Zhao et al. Apatinib + PTX/POF for taxane-resistant advanced GC

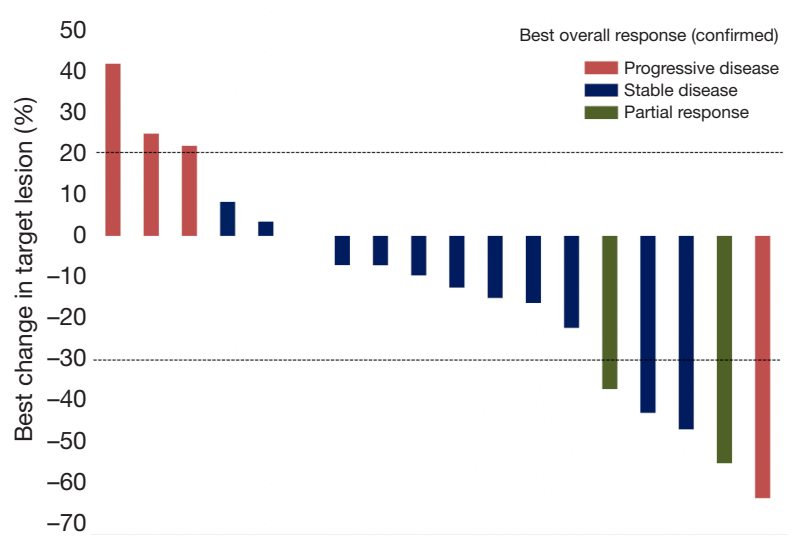

Figure 1 Waterfall plot of best change in tumor dimension.

\section{Efficacy}

No patient had a CR, two patients had a PR, 12 patients had stable disease (SD), and four patients had progressive disease (PD). The ORR was $11.1 \%$ (95\% CI: 1.4-34.7\%) and the DCR was $77.8 \%$ (95\% CI: 52.4-93.6\%) (Table 2). Figure 1 presents the waterfall plot of best change in tumor dimension. The median PFS was 3.5 (95\% CI: 1.9-5.1) months, the median OS and TTP were 4.7 (95\% CI: 2.0-7.3) and 4.2 (95\% CI 0.562-7.838) months, respectively (Figure 2).

AEs

Table 3 presents the AEs. All 20 (100\%) patients had AEs. The most common AEs (in $>40 \%$ of the patients) were neutropenia (80\%), leukopenia (75\%), anemia (70\%), hypoalbuminemia (55\%), hypertension $(50 \%)$, fatigue $(50 \%)$, hypokalemia $(45 \%)$, increased $\gamma$-glutamyltransferase $(40 \%)$, loss of appetite $(40 \%)$, increased aspartate aminotransferase (40\%), and increased lactate dehydrogenase $(40 \%)$. The most common grade 3-4 AEs included neutropenia (30\%), leukopenia (30\%), hypertension $(30 \%)$, anemia (20\%), fatigue (15\%), and hypokalemia $(15 \%)$. A total of $17(85 \%)$ had apatinib treatment-emergent AEs (TEAEs), and 18 (90\%) had chemotherapy TEAEs. The most common grade 3-4 TEAEs were neutropenia, leukopenia, hypertension, and anemia. Four patients had grade 5 AEs and died of pulmonary infection, pneumonia, incomplete intestinal obstruction, and disease progression, respectively. Patients who died of interstitial pneumonia were associated with apatinib treatment, other deaths were not related to 

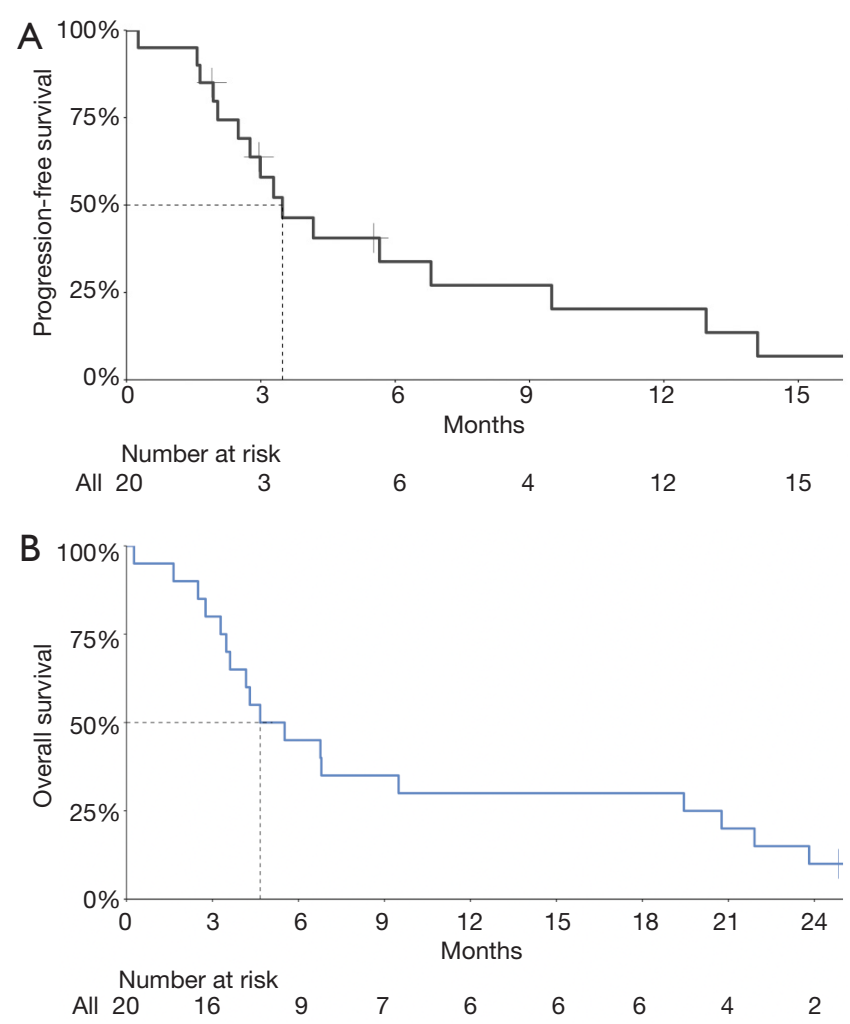

Figure 2 Kaplan-Meier plot of progression-free survival (A) and overall survival (B). The median progression-free survival was 3.5 (95\% CI: 1.9-5.1) months. The median overall survival was 4.7 (95\% CI: 2.0-7.3) months.

treatment.

\section{Discussion}

Treatment for patients with chemotherapy-refractory advanced gastric cancer remains an unmet need. Therefore, this single-center, open-labeled, single-arm, exploratory study aimed to investigate the efficacy and safety of apatinib in combination with POF or PTX alone in patients with taxane-resistant advanced gastric cancer. The results showed that the ORR was $11.1 \%$ (95\% CI: $1.4-34.7 \%)$, the DCR was $77.8 \%$ (95\% CI: 52.4-93.6\%), the median PFS was 3.5 (95\% CI: 1.9-5.1) months, and the median OS was 4.7 (95\% CI: 2.0-7.3) months. All patients had AEs, and the main grade 3-4 TEAEs were neutropenia, leukopenia, hypertension, and anemia. Those results suggest that apatinib combined with PTX or POF might be effective and tolerable in patients with chemotherapy-refractory gastric cancer.
There are similar studies in which apatinib versus placebo, apatinib in combination with chemotherapy versus chemotherapy for patients with chemotherapy-refractory advanced or metastatic adenocarcinoma of the stomach or gastroesophageal junction. Apatinib alone vs. placebo improved the PFS (2.6 vs. 1.8 months) and OS (6.5 vs. 4.7 months) of patients with advanced gastric cancer after failure to at least two lines of treatment, with a better ORR and DCR $(2.84 \%$ vs. $0 \%, 42.05 \%$ vs. $8.79 \%)$ in favour of apatinib $(8,9)$. This is supported by two registry studies $(13,14)$, real-world studies $(15,16)$, and a phase IV trial $(17)$. Guo et al. (10) showed that apatinib in combination with chemotherapy was effective in the treatment of advanced or metastatic chemotherapy-refractory gastric cancer, and had higher PFS (8.5 vs. 7.0 months) and DCR (58.4\% vs. $41.9 \%)$ than that of chemotherapy alone, but ORR was similar in two groups. However, the study was heterogeneous in terms of chemotherapy regimens, including FU-based regimens, PTX-based regimens, FU + PTX/DOC + platinum regimens and irinotecan-based regimens. It was difficult to evaluate the impact of certain regimen.

The present study showed that apatinib combined with PTX-based regimens, either PTX alone or POF, was effective for the control of advanced chemotherapyrefractory gastric cancer. The therapeutic mechanism might be that apatinib enhanced the sensitivity of gastric cancer to PTX. The proliferation inhibition and apoptosis rate were significantly strengthened in a variety of gastric cancer cell lines when apatinib was combined with PTX. Apatinib also enhanced PTX-mediated antitumor efficacy in transparent Casper zebrafish early-stage embryos and gastric cancer xenografts, and significantly reduced tumor growth, tumor size and microvessel density $(18,19)$. Furthermore, apatinib has been shown to reverse the resistance to PTX by affecting cell membrane-bound ATP-binding cassette $(\mathrm{ABC})$ transporters, which extrude different chemotherapeutic agents out of the cancer cells. Apatinib reverses ABCB1- and ABCG2-mediated multidrug resistance by inhibiting their transport function $(20,21)$. Nevertheless, the exact mechanisms were not the focus of the present exploratory study, and additional studies will be necessary to examine this point.

In addition, Ando et al. (22) showed that weekly PTX showed some efficacy in DOC-resistant patients with advanced gastric cancer. The patients who received PTX after refractory to DOC-based chemotherapy achieved a response rate of $14.5 \%$, suggested only partial crossresistance between DOC and PTX. Future studies should 
Table 3 AEs and treatment-related AEs $(\mathrm{n}=20)$

\begin{tabular}{|c|c|c|c|c|c|c|c|c|c|}
\hline & \multicolumn{3}{|c|}{$\mathrm{AE}, \mathrm{n}[\%]$} & \multicolumn{3}{|c|}{ Apatinib TEAE, n [\%] } & \multicolumn{3}{|c|}{ Chemotherapy TEAE, n [\%] } \\
\hline Any grade $A E$ & $20[100]$ & 17 [85] & $4[20]$ & 17 [85] & $12[60]$ & $1[5]$ & $18[90]$ & $11[55]$ & 0 \\
\hline Decreased neutrophil count & $16[80]$ & $6[30]$ & 0 & $6[30]$ & $2[10]$ & 0 & $16[80]$ & $6[30]$ & 0 \\
\hline Decreased leukocyte count & 15 [75] & $6[30]$ & 0 & 7 [35] & $3[15]$ & 0 & $14[70]$ & 5 [25] & 0 \\
\hline Hypoalbuminemia & $11[55]$ & 0 & 0 & $1[5]$ & 0 & 0 & 0 & 0 & 0 \\
\hline Hypertension & $10[50]$ & $6[30]$ & 0 & $7[35]$ & $3[15]$ & 0 & $2[10]$ & $1[5]$ & 0 \\
\hline Fatigue & $10[50]$ & $3[15]$ & 0 & $8[40]$ & $3[15]$ & 0 & $8[40]$ & $3[15]$ & 0 \\
\hline Hypokalemia & 9 [45] & $3[15]$ & 0 & $1[5]$ & 0 & 0 & $1[5]$ & 0 & 0 \\
\hline Increased aspartate aminotransferase & $8[40]$ & $1[5]$ & 0 & $2[10]$ & $1[5]$ & 0 & $4[20]$ & $1[5]$ & 0 \\
\hline Increased lactate dehydrogenase & $8[40]$ & 0 & 0 & $2[10]$ & 0 & 0 & 5 [25] & 0 & 0 \\
\hline Decreased platelet count & $7[35]$ & $2[10]$ & 0 & $1[5]$ & 0 & 0 & $4[20]$ & $1[5]$ & 0 \\
\hline Increased alanine aminotransferase & $6[30]$ & $1[5]$ & 0 & $2[10]$ & $1[5]$ & 0 & 5 [25] & $1[5]$ & 0 \\
\hline Proteinuria & $6[30]$ & 0 & 0 & $5[25]$ & 0 & 0 & $2[10]$ & 0 & 0 \\
\hline Diarrhea & $6[30]$ & 0 & 0 & $4[20]$ & 0 & 0 & $3[15]$ & 0 & 0 \\
\hline Increased alkaline phosphatase & $6[30]$ & $1[5]$ & 0 & $3[15]$ & 0 & 0 & $4[20]$ & 0 & 0 \\
\hline Hypocalcemia & $5[25]$ & 0 & 0 & $1[5]$ & 0 & 0 & $1[5]$ & 0 & 0 \\
\hline Increased blood bilirubin & $4[20]$ & $1[5]$ & 0 & $2[10]$ & $1[5]$ & 0 & $2[10]$ & $1[5]$ & 0 \\
\hline Hypophosphatemia & $3[15]$ & $2[10]$ & 0 & 0 & 0 & 0 & 0 & 0 & 0 \\
\hline Dysphonia & $3[15]$ & 0 & 0 & $3[15]$ & 0 & 0 & $1[5]$ & 0 & 0 \\
\hline Abdominal distention & $3[15]$ & 0 & 0 & 0 & 0 & 0 & 0 & 0 & 0 \\
\hline Cough & $3[15]$ & 0 & 0 & 0 & 0 & 0 & 0 & 0 & 0 \\
\hline Prolonged clotting time & $3[15]$ & 0 & 0 & 0 & 0 & 0 & 0 & 0 & 0 \\
\hline Decreased blood urea & $3[15]$ & 0 & 0 & 0 & 0 & 0 & 0 & 0 & 0 \\
\hline Palmar-Plantar Erythrodysesthesia Syndrome & $3[15]$ & $1[5]$ & 0 & $2[10]$ & $1[5]$ & 0 & $2[10]$ & 0 & 0 \\
\hline Increased carcinoembryonic antigen & $2[10]$ & 0 & 0 & 0 & 0 & 0 & 0 & 0 & 0 \\
\hline Hypoproteinemia & $2[10]$ & 0 & 0 & 0 & 0 & 0 & 0 & 0 & 0 \\
\hline Hyponatremia & $2[10]$ & 0 & 0 & 0 & 0 & 0 & 0 & 0 & 0 \\
\hline
\end{tabular}

Table 3 (continued) 
Table 3 (continued)

\begin{tabular}{|c|c|c|c|c|c|c|c|c|c|}
\hline & \multicolumn{3}{|c|}{$\mathrm{AE}, \mathrm{n}[\%]$} & \multicolumn{3}{|c|}{ Apatinib TEAE, n [\%] } & \multicolumn{3}{|c|}{ Chemotherapy TEAE, n [\%] } \\
\hline Nausea & $2[10]$ & 0 & 0 & $1[5]$ & 0 & 0 & $1[5]$ & 0 & 0 \\
\hline Pulmonary infection & $2[10]$ & 0 & $1[5]$ & 0 & 0 & 0 & 0 & 0 & 0 \\
\hline Pneumonia & $2[10]$ & 0 & $1[5]$ & $1[5]$ & 0 & $1[5]$ & 0 & 0 & 0 \\
\hline Myalgia & $2[10]$ & 0 & 0 & 0 & 0 & 0 & 0 & 0 & 0 \\
\hline Mouth ulcers & $2[10]$ & 0 & 0 & $1[5]$ & 0 & 0 & $2[10]$ & 0 & 0 \\
\hline Epigastric discomfort & $2[10]$ & 0 & 0 & 0 & 0 & 0 & $1[5]$ & 0 & 0 \\
\hline Insomnia & $2[10]$ & 0 & 0 & $1[5]$ & 0 & 0 & $1[5]$ & 0 & 0 \\
\hline Peripheral nerve injury & $2[10]$ & 0 & 0 & $1[5]$ & 0 & 0 & $1[5]$ & 0 & 0 \\
\hline Dizziness & $2[10]$ & 0 & 0 & 0 & 0 & 0 & 0 & 0 & 0 \\
\hline Increased blood uric acid & $2[10]$ & 0 & 0 & 0 & 0 & 0 & $2[10]$ & 0 & 0 \\
\hline Hematuria & $2[10]$ & 0 & 0 & $1[5]$ & 0 & 0 & $1[5]$ & 0 & 0 \\
\hline Increased blood fibrinogen & $2[10]$ & 0 & 0 & 0 & 0 & 0 & 0 & 0 & 0 \\
\hline Hypercoagulable state & $2[10]$ & 0 & 0 & 0 & 0 & 0 & 0 & 0 & 0 \\
\hline Limb pain & $2[10]$ & $1[5]$ & 0 & $2[10]$ & $1[5]$ & 0 & $2[10]$ & $1[5]$ & 0 \\
\hline Increased tumor markers & $2[10]$ & 0 & 0 & 0 & 0 & 0 & 0 & 0 & 0 \\
\hline Oral pain & $1[5]$ & $1[5]$ & 0 & $1[5]$ & $1[5]$ & 0 & 0 & 0 & 0 \\
\hline Disease progression & $1[5]$ & 0 & $1[5]$ & 0 & 0 & 0 & 0 & 0 & 0 \\
\hline Drug hypersensitivity & $1[5]$ & $1[5]$ & 0 & 0 & 0 & 0 & $1[5]$ & $1[5]$ & 0 \\
\hline
\end{tabular}

$\mathrm{AE}$, adverse events; TEAE, treatment emergent adverse events.

examine the combination of apatinib with PTX $v$ s. weekly PTX in the context of DOC-resistance.

This study showed that apatinib, in combination with PTX or POF, was tolerable. All patients had AEs, but $85 \%$ had apatinib TEAEs, and $90 \%$ had chemotherapy TEAEs. The most common AEs were hematological (neutropenia, leukopenia, and anemia), liver (hypoalbuminemia, increased $\gamma$-glutamyltransferase, increased aspartate aminotransferase, and increased alanine aminotransferase), hypertension, fatigue, hypokalemia, and loss of appetite. Those most common AEs were in accordance with the known safety features of PTX, POF, and apatinib, since all three treatments could induce those AEs $(1,7,23,24)$. Previous studies have shown that patients with gastric cancer could tolerate apatinib in combination with PTX- and platinumbased chemotherapy $(10,18)$, supporting the present study. 


\section{Page 8 of 9}

However, the incidence of common AEs in this study was higher than that in the study of apatinib combined with chemotherapy (10). One case of apatinib-related death occurred. This patient did not inform the investigator in the early stage of pneumonia, resulting in delayed treatment time, and was in an incurable state in the hospital, which eventually leading to the patient's death.

Of course, the present study has limitations. It was only a small exploratory trial, without a comparison group, and without power analysis. Hence, the efficacy and safety of the treatment will have to be confirmed in future clinical trials.

In conclusion, this study preliminarily showed that apatinib combined with PTX or POF might be effective and tolerable in the treatment of chemotherapy-refractory advanced gastric cancer.

\section{Acknowledgments}

Funding: This work was supported by the Fujian Provincial Health Technology Project (2016-CX-12), Natural Science Foundation of Fujian Province (2018J01271, 2019J01199), and Beijing Xisike Clinical Oncology Research Foundation (Grant No: Y-HR2016-055).

\section{Footnote}

Reporting Checklist: The authors have completed the TREND reporting checklist. Available at http://dx.doi. org/10.21037/atm-20-5841

Data Sharing Statement: Available at http://dx.doi. org/10.21037/atm-20-5841

Conflicts of Interest: All authors have completed the ICMJE uniform disclosure form (available at http://dx.doi. org/10.21037/atm-20-5841). Dr. Lin reports grants from the Fujian Provincial Health Technology Project, grants from Natural Science Foundation of Fujian Province, grants from Beijing Xisike Clinical Oncology Research Foundation, during the conduct of the study; Dr. Zhao reports grants from Natural Science Foundation of Fujian Province, during the conduct of the study. The other authors have no conflicts of interest to declare.

Ethical Statement: The authors are accountable for all aspects of the work in ensuring that questions related to the accuracy or integrity of any part of the work are appropriately investigated and resolved. This study is
Zhao et al. Apatinib + PTX/POF for taxane-resistant advanced GC

performed in accordance with the Declaration of Helsinki (as revised in 2013), Good Clinical Practice, and related laws. The study protocol was approved by the ethics committee of Fujian Cancer Hospital. The study was registered (ClinicalTrials.gov \#NCT02697838). All patients provided a signed informed consent form before any study procedure.

Open Access Statement: This is an Open Access article distributed in accordance with the Creative Commons Attribution-NonCommercial-NoDerivs 4.0 International License (CC BY-NC-ND 4.0), which permits the noncommercial replication and distribution of the article with the strict proviso that no changes or edits are made and the original work is properly cited (including links to both the formal publication through the relevant DOI and the license). See: https://creativecommons.org/licenses/by-nc-nd/4.0/.

\section{References}

1. NCCN Clinical Practice Guidelines in Oncology (NCCN Guidelines). Gastric Cancer. Version 2.2020. Fort

Washington: Natiocal Comprehensive Cancer Network; 2020.

2. Van Cutsem E, Sagaert X, Topal B, et al. Gastric cancer. Lancet 2016;388:2654-64.

3. Bray F, Ferlay J, Soerjomataram I, et al. Global cancer statistics 2018: GLOBOCAN estimates of incidence and mortality worldwide for 36 cancers in 185 countries. CA Cancer J Clin 2018;68:394-424.

4. Smyth EC, Verheij M, Allum W, et al. Gastric cancer: ESMO Clinical Practice Guidelines for diagnosis, treatment and follow-up. Ann Oncol 2016;27:v38-v49.

5. Fagin JA, Wells SA Jr. Biologic and Clinical Perspectives on Thyroid Cancer. N Engl J Med 2016;375:2307.

6. Lin R, Chen Y, Zhu J, et al. POF (paclitaxel plus FOLFOX) versus IP PAC (intraperitoneal paclitaxel plus FOLFOX) versus FOLFOX as a first-line treatment in advanced gastric cancer (AGC): Update from a multicenter, randomized phase II trial, FNF-004 trial. J Clin Oncol 2019;37:abstr 4035.

7. Scott LJ. Apatinib: A Review in Advanced Gastric Cancer and Other Advanced Cancers. Drugs 2018;78:747-58.

8. Li J, Qin S, Xu J, et al. Randomized, Double-Blind, Placebo-Controlled Phase III Trial of Apatinib in Patients With Chemotherapy-Refractory Advanced or Metastatic Adenocarcinoma of the Stomach or Gastroesophageal Junction. J Clin Oncol 2016;34:1448-54. 
9. Li J, Qin S, Xu J, et al. Apatinib for chemotherapyrefractory advanced metastatic gastric cancer: results from a randomized, placebo-controlled, parallel-arm, phase II trial. J Clin Oncol 2013;31:3219-25.

10. Guo Y, Tang J, Huang XE, et al. Efficacy and toxicity of apatinib combined with or without chemotherapy for patients with advanced or metastatic chemotherapyrefractory gastric adenocarcinoma: A prospective clinical study. Medicine (Baltimore) 2019;98:e13908.

11. Schwartz LH, Litiere S, de Vries E, et al. RECIST 1.1-Update and clarification: From the RECIST committee. Eur J Cancer 2016;62:132-7.

12. U.S. Department of Health and Human Services. National Institutes of Health. National Cancer Institutes. Common Terminology Criteria for Adverse Events (CTCAE) Version 4.0. Available online: https://evs.nci.nih.gov/ ftp1/CTCAE/CTCAE_4.03/CTCAE_4.03_2010-06-14_ QuickReference_8.5x11.pdf. Accessed 05-21-2020. 2009.

13. Feng J, Shen B, He Z, et al. Real world data about clinical efficacy and safety of apatinib in the treatment of advanced gastric cancer. Ann Oncol 2017;28:v209-68.

14. Bai C, Zhong D, Zhang R, et al. Prospective, multicenter, non-interventional and registry clinical study of apatinib in patients with advanced gastric cancer. J Clin Oncol 2018;36:abstr 137.

15. Zhu H, Sun X, Zhou Q. Clinical study of apatinib combined chemotherapy in the treatment of advanced gastric cancer. Chin J Clin Oncol 2016;8:394-6.

16. Wang B, Song LJ, Niu PY. Clinical efficacy of apatinib in treatment of advanced gastric cancer. World Chin J Digestol 2016;24:759-64.

17. Deng W, Qin S, Li J, et al. Initial dose of apatinib in

Cite this article as: Zhao S, Fan N, Li H, Liu J, Huang F, Chen Y, Zhou M, Yu J, Lin R. Apatinib combined with paclitaxel-based chemotherapy in patients with taxane-resistant advanced gastric cancer: a single-arm exploratory study. Ann Transl Med 2020;8(19):1233. doi: 10.21037/atm-20-5841
Chinese patients with chemotherapy-refractory advanced or metastatic adenocarcinoma or gastroesophageal junction in third- and later-line setting: $500 \mathrm{mg}$ or $850 \mathrm{mg}$ ? J Clin Oncol 2018;36:abstr 35.

18. Xu Z, Hu C, Chen S, et al. Apatinib enhances chemosensitivity of gastric cancer to paclitaxel and 5-fluorouracil. Cancer Manag Res 2019;11:4905-15.

19. Fathi Maroufi N, Rashidi MR, Vahedian V, et al. Therapeutic potentials of Apatinib in cancer treatment: Possible mechanisms and clinical relevance. Life Sci 2020;241:117106.

20. Zhang Q, Song Y, Cheng X, et al. Apatinib Reverses Paclitaxel-resistant Lung Cancer Cells (A549) Through Blocking the Function of ABCB1 Transporter. Anticancer Res 2019;39:5461-71.

21. Mi Y-J, Liang Y-J, Huang H-B, et al. Apatinib (YN968D1) reverses multidrug resistance by inhibiting the efflux function of multiple ATP-binding cassette transporters. Cancer research 2010;70:7981-91.

22. Ando T, Hosokawa A, Kajiura S, et al. Efficacy of weekly paclitaxel in patients with advanced gastric cancer refractory to docetaxel-based chemotherapy. Gastric Cancer 2012;15:427-32.

23. Lin RB, Fan NF, Guo ZQ, et al. A phase II study of 5 -fluorouracil/leucovorin in combination with paclitaxel and oxaliplatin as first-line treatment for patients with advanced gastric cancer. J Chemother 2008;20:744-8.

24. Dai X, Zhang X, Wang C, et al. Paclitaxel/oxaliplatin/ fluorouracil (TOF) regimen versus S-1/oxaliplatin (SOX) regimen for metastatic gastric cancer patients. Oncotarget 2017;8:30495-501. 